\title{
MCAO near-IR photometry of the globular cluster NGC 6388: MAD observations in crowded fields ${ }^{\star}$
}

\author{
A. Moretti ${ }^{1}$, G. Piotto ${ }^{2}$, C. Arcidiacono ${ }^{1}$, A. P. Milone ${ }^{2}$, R. Ragazzoni ${ }^{1}$, R. Falomo ${ }^{1}$, J. Farinato ${ }^{1}$, L. R. Bedin ${ }^{3}$, \\ J. Anderson ${ }^{3}$, A. Sarajedini ${ }^{4}$, A. Baruffolo ${ }^{1}$, E. Diolaiti ${ }^{5}$, M. Lombini ${ }^{5}$, R. Brast ${ }^{6}$, R. Donaldson $^{6}$, J. Kolb $^{6}$, \\ E. Marchetti ${ }^{6}$, and S. Tordo 6
}

\author{
1 INAF - Osservatorio Astronomico di Padova, Vicolo dell'Osservatorio 5, 35122 Padova, Italy \\ e-mail: alessia.moretti@unipd.it \\ 2 Dipartimento di Astronomia - Università di Padova, Vicolo dell’Osservatorio 3, 35122 Padova, Italy \\ 3 Space Telescope Science Institute, 3800 San Martin Drive, Baltimore, MD 21218, USA \\ 4 Department of Astronomy, University of Florida, Gainesville 32611, USA \\ 5 INAF - Osservatorio Astronomico di Bologna, via Ranzani 1, 40127 Bologna, Italy \\ ${ }^{6}$ European Southern Observatory, Karl-Schwarzschild-Strasse 2, 85748 Garching, Germany \\ Received 30 July 2008 / Accepted 6 October 2008
}

\section{ABSTRACT}

\begin{abstract}
Context. Deep photometry of crowded fields, such as Galactic globular clusters, is severely limited by the resolution of ground-based telescopes. On the other hand, the Hubble Space Telescope does not have the near-infrared (NIR) filters needed to allow large color baselines.

Aims. In this work we demonstrate how ground based observations can reach the required resolution when using Multi-Conjugated Adaptive Optic (MCAO) devices in the NIR, such as the experimental infrared camera (MAD) available on the VLT. This is particularly important since these corrections are planned to be available on all ground-based telescopes in the near future.

Methods. We demonstrate this by combining the infrared photometry obtained by MAD/VLT with ACS/HST optical photometry of our scientific target, the bulge globular cluster NGC 6388, in which we imaged two fields. In particular, we constructed colormagnitude diagrams with an extremely wide color baseline in order to investigate the presence of multiple stellar populations in this cluster.

Results. From the analysis of the external field, observed with better seeing conditions, we derived the deepest optical-NIR CMD of NGC 6388 to date. The high-precision photometry reveals that two distinct sub-giant branches are clearly present in this cluster. We also use the CMD from the central region to estimate the distance $\left[(m-M)_{\circ}=15.33\right]$ and the reddening $(E(B-V)=0.38)$ for this cluster. We estimate the age to be $(\sim 11.5 \pm 1.5 \mathrm{Gyr})$. The large relative-age error reflects the bimodal distribution of the SGB stars. Conclusions. This study clearly demonstrates how MCAO correction in the NIR bands implemented on ground based telescopes can complement the high-resolution optical data from HST.
\end{abstract}

Key words. stars: Hertzsprung-Russell (HR) and C-M diagrams - galaxy: globular clusters: general - stars: late-type

\section{Introduction}

NGC 6388 and NGC 6441 are among the most intriguing Galactic globular clusters (GCs). They are both located in the Galactic bulge. Early studies of their integrated light by Rich et al. (1993) revealed an anomalous UV-excess. Both clusters are metal-rich $([\mathrm{Fe} / \mathrm{H}] \sim-0.6$ and $\sim-0.5$, respectively) and should therefore possess a red horizontal branch (HB), with no hot blue HB stars. However, deep high-resolution images obtained by the Hubble Space Telescope (HST) revealed that both clusters exhibit a very long tail of blue HB stars that explains the UV excess (Rich et al. 1997). In addition, the HBs of NGC 6388 and NGC 6441 have a sloped appearance (Raimondo et al. 2002; Busso et al. 2007), which can not be reproduced by stellar evolution models with the clusters' known metallicities and typical GC abundance ratios (see also Catelan et al. 2006, for a detailed discussion). When compared with 47 Tuc, NGC 6388 shows a broader color-magnitude diagram (CMD), both in the turnoff region and along the Red Giant Branch (RGB), which is at least in

* Based on observations collected at the European Southern Observatory, Chile, as part of MAD Guaranteed Time Observations, and on NASA/ESA Hubble Space Telescope observations (GO-10775). part attributed to the presence of differential reddening (Busso et al. 2007)

What then is responsible for the long blue tail of hot stars in these clusters? The solution to this problem, which might be the most extreme manifestation of the long-standing secondparameter problem, has yet to be established. Some resolution might come from the observation that the most massive GCs of our Galaxy ( $\omega$ Cen, NGC 2808, NGC 1851) possess CMDs which split into multiple evolutionary sequences, either in the MS region (NGC 2808, Piotto et al. 2007), or in the Subgiant Branch region (NGC 1851, Milone et al. 2008), or in the MS, $\mathrm{SGB}$, and RGB regions ( $\omega$ Cen, Lee et al. 1999; Pancino et al. 2000; Bedin et al. 2004; Villanova et al. 2007).

The recent evidence (Piotto 2008) that the SGB of NGC 6388 is split into two distinct branches gives further support to the possibility that this cluster may also host more than one stellar population.

Ree et al. (2002) demonstrated that the peculiarities of NGC 6388's CMD (i.e. the broad RGB and the bimodal HB) can be explained by allowing a double generation of stars with a mild age-metallicity relation. Alternatively, the second generation of stars could be formed from material already enriched in 
Table 1. Observation log.

\begin{tabular}{lcccccccc}
\hline \hline Name & Night & Filter & DIT & NDIT & $T_{\text {exp }}$ & $\langle F W H M\rangle$ & Seeing & $m_{F 606 W}$ (AO stars) \\
\hline NGC 6388-a & $26 / 9 / 2007$ & $K_{\mathrm{s}}$ & 10 & 4 & 3000 & 0.27 & 1.76 & $13.6-13.9-14.0-14.8-15.1$ \\
NGC 6388-b & $27 / 9 / 2007$ & $K_{\mathrm{s}}$ & 10 & 24 & 5040 & 0.15 & 0.46 & $15.0(2)-15.6-15.7-16.3$ \\
\hline
\end{tabular}

CNO-cycle products by AGB stars, and could therefore have enhanced helium abundance (D'Antona et al. 2002; D'Antona \& Caloi 2004). We note that for helium to explain the blue tail, it cannot involve simple deep mixing, but must involve an actual abundance variation. Such a helium-enhanced population could have an age difference from the first generation of only a few hundred million years, not measurable on the CMD. The Yoon et al. (2008) simulations of the observed CMDs of NGC 6388 and NGC 6441 confirm that the only mechanism able to produce a tilted HB and the observed RR Lyr properties is a selfenrichment scenario; however, they find a lower helium content for the enhanced population than do Caloi \& D'Antona (2007). Spectroscopic analysis by Carretta et al. (2007) confirms that the BHB can be caused by this self-enriched helium-enhancement; they also rule out large age differences between the generations.

A strong indicator of the presence of multiple generation of stars with different helium content in NGC 6388 would be the discovery of the presence of multiple main-sequence populations (see Piotto et al. 2007). However, since the cluster is located in the Galactic bulge, the strong contamination by field stars and the differential reddening make this investigation rather complicated.

In principle, near-infrared (NIR) observations should improve our ability to distinguish different stellar generations in NGC 6388, because of the reduced effect of differential reddening and because of the larger color baseline obtained when coupling the NIR observations with optical ones. Theoretical models predict, for example, that sequences characterized by a different helium content would appear well separated, and this is easier to distinguish with a larger color baseline. In the case of NGC 6388 a difference of 0.15 in helium content (as suggested by D'Antona \& Caloi 2008) would cause a color split of 0.13 mag in $m_{F 606 W}-K_{\mathrm{S}}$ which corresponds to 0.04 in $(B-V)$. On the other hand the broadening effect due to the effects of differential reddening can be minimized as in Sarajedini et al. (2007) and Milone et al. (2008), thus making this combination of passbands very appealing. In addition to exploring the main sequence, the NIR observations would also allow us to verify that the presence of the double SGB identified by Piotto (2008) is not an artifact of differential reddening. Moreover, theoretical isochrones show that the chosen optical-NIR color is much more sensitive to age differences (1 Gyr corresponding to $m_{F 606 W}-K_{\mathrm{s}} \sim 0.03$ and $\left.B-V \sim 0.01\right)$ than pure optical colors.

For all the above reasons, we decided to observe this massive bulge GC using the Multi-conjugated Adaptive optics Demonstrator (MAD) on the VLT in the hope of obtaining accurate photometry in order to explore the evidence for multiple populations in this cluster. With this goal in mind, we also tried to test MAD capabilities under two different conditions. We observed two fields in NGC 6388: one located at the center of the cluster, where we can sample a large number of stars, though crowding severely affects the images (NGC 6388-a), and a second less dense field (NGC 6388-b), located 110 arcsec away from the center. The external field was used as a test of MAD performance in terms of high-precision photometry.

\section{Observations and analysis}

Near-IR observations were carried out with the MultiConjugated Adaptive Optics Demonstrator (MAD) mounted at the VLT (Marchetti et al. 2007). MAD is a prototype Multi Conjugated Adaptive Optics (MCAO) system which has been designed to test in the laboratory and on the sky the feasibility of different MCAO image reconstruction and correction techniques, in view of future applications with the 2nd Generation VLT and E-ELT instrumentation.

The MAD imager is a 1 arcmin field-of-view (FoV) infrared camera scanning a 2 arcmin FoV (round). The CCD has $2048 \times 2048$ pixels with a scale of 0.028 arcsec/pixel (Marchetti et al. 2006).

The wavefront sensor that we used is a Layer Oriented (LO) multi pyramid sensor (Ragazzoni 1996; Ragazzoni et al. 2000; Vernet et al. 2005; Arcidiacono et al. 2006, 2007) that can use from 3 to 8 "faint" $(V<18)$ stars, for which the integrated light reaches $V=13$. The stars can be placed anywhere in the telescope FoV. The LO technique has a great advantage, with respect to the Star Oriented (SO) one, because it offers the possibility of using fainter stars (see Ragazzoni \& Farinato 1999; Ghedina et al. 2003, for a detailed discussion).

Observations of the two fields in NGC 6388 were made in September 2007 using the full MCAO capability. These were part of a set of science observations aimed at verifying the capability of MAD at VLT (Gullieuszik et al. 2008; Falomo et al. 2008). In Table 1 we show the observation log: the first field (labeled NGC 6388-a) is located at the center of the cluster $(17: 36: 17.86,-44: 44: 05.60)$, and the second one $(\mathrm{NGC} 6388-\mathrm{b})$ at $\mathrm{RA}(\mathrm{J} 2000.0)=17: 36: 22.86, \operatorname{Dec}(\mathrm{J} 2000.0)=$ $-44: 45: 35.53$ (see Fig. 1). The last column indicates the $V$ magnitude of the stars used to perform the AO correction.

It is remarkable that the reference stars used for the MCAO correction in the case of NGC 6388-b are significantly fainter than the ones for NGC 6388-a and, in general, much fainter than the ones usually adopted for the Star Oriented Wavefront Sensor. In spite of this, the outcome is significantly better, as the initial seeing is significantly better, although well within the median seeing of the VLT site.

In both cases, dedicated sky exposures were taken at an appropriate distance from the scientific frame. For these images the background subtraction has been performed taking into account the sky level and shape determined from the offset frames.

The data-reduction process started with the trimming of the images in order to exclude from further analysis the overscan region. Flat-field images were taken on the sky at the beginning of each night in the $K_{\mathrm{s}}$ filter (centered at $2.15 \mu$ ). After excluding the bad flats we combined them into a median frame that was used to correct the scientific frames. Flat-field images were used to produce the appropriate bad pixel mask as well.

Each scientific frame was then corrected with the flat-field, and bad pixels removed using the constructed mask. After having verified that the sky variations during the observations were negligible, we decided to use one single sky image to correct all scientific frames. 

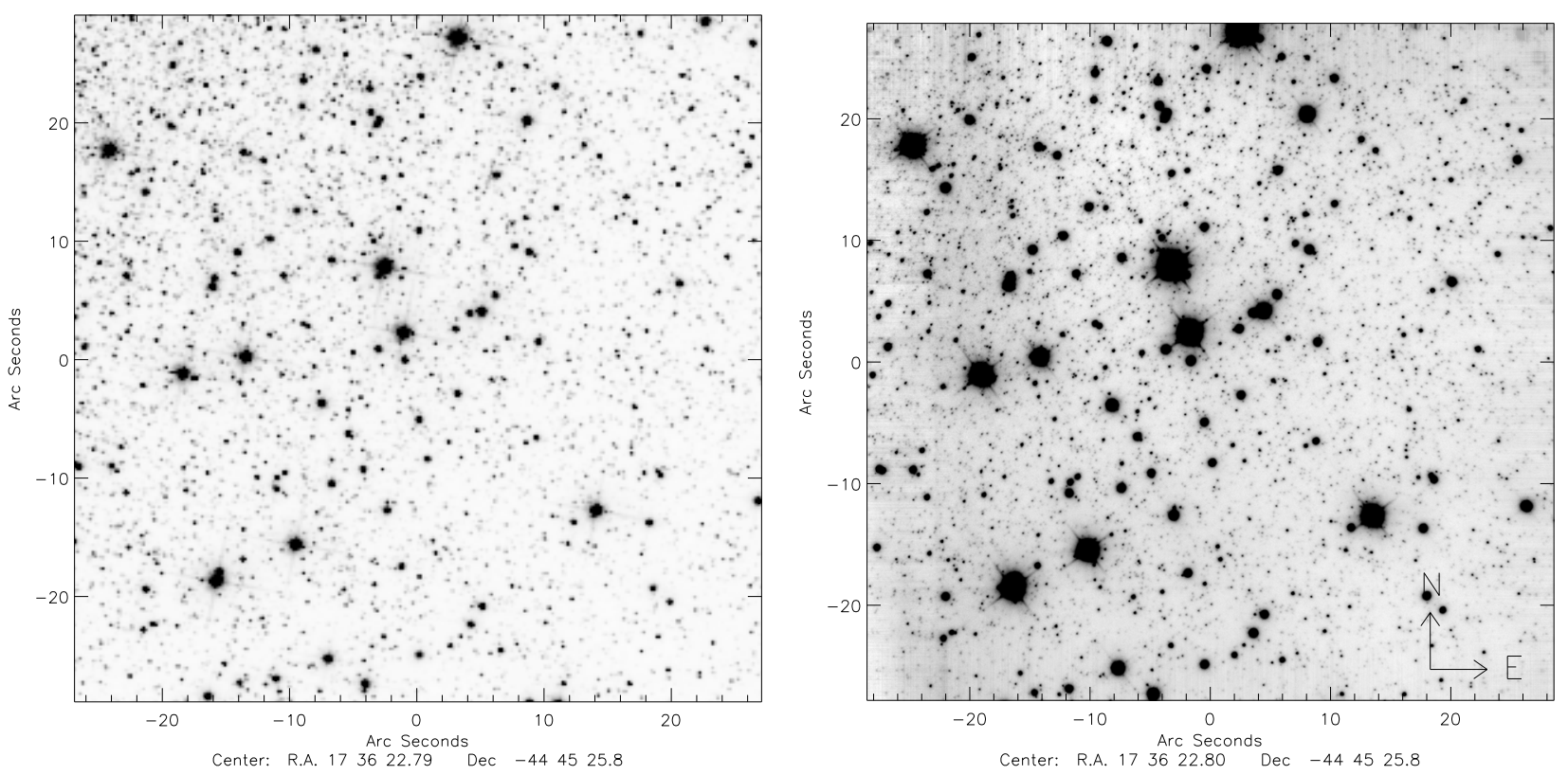

Fig. 1. The external field, NGC 6388 b, imaged by ACS/HST in the F814W filter (left) and by MAD/VLT (right). Exposure times are $1790 \mathrm{~s}$ and $240 \mathrm{~s}$, respectively.

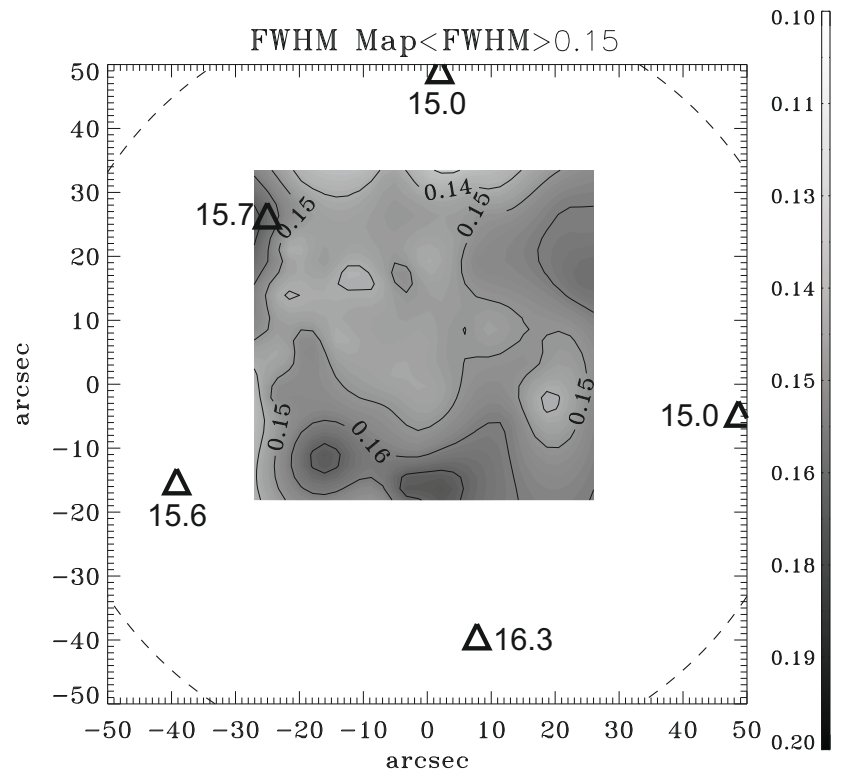

Fig. 2. FWHM map of the field of NGC 6388-b that was observed with MAD. Triangles represent the stars used to perform the AO correction. Contours are drawn and labelled according to the FWHM measured on observed stars. Magnitudes for AO stars are given in $m_{F 606 W}$.

In the case of crowded fields (such as globular clusters) it is normal practice to use offset positions to determine the mean level and the shape of the sky/background correction by making the median of these sky frames. In particular, when possible we used the ones closer in time to the scientific images. The final sky/background frame has been normalized to the median counts of the scientific frame before being subtracted.

We finally measured the overall stellar FWHM over the FoV, to check the performance of the AO correction. Figure 2 shows, as an example, the FWHM map obtained for the field NGC 6388b. Note the strong improvement of the seeing due to the AO correction (column seven of Table 1) with respect to the ESO DIMM monitor seeing (column eight of Table 1).
The standard star FS5, taken from UKIRT Faint Standard catalogue (Hawarden et al. 2001), was observed, and then used to calibrate the photometry. Unfortunately, the observation timing and the need to manually operate the filters did not allow us to take frames in other filters (such as $J$ or $H$ ), so that no color dependence of the photometric zeropoint could be determined.

Our dataset in the central region consists of 75 images with DIT $=10 \mathrm{~s}, \mathrm{NDIT}=4$ that are characterized by an average $F W H M$ of 0.27 arcsec. As for the external field, we have 21 images with DIT $=10 \mathrm{~s}$, NDIT $=24$, characterized by a much smaller stellar FWHM (on average 0.15 arcsec).

\subsection{Photometry}

We adopted two different approaches for the photometric analysis of our observations. One consisted of making an average image out of the observed ones (excluding the ones with the worst seeing), and performing photometry with DAOPHOT/ALLSTAR (Stetson 1987) on this image. This approach was used for the central field, where we selected the exposures with a stellar $F W H M$ smaller than $0.5 \operatorname{arcsec}(25$ out of 75), and combined them into a single one with a total exposure time of $1000 \mathrm{~s}$.

The second approach consists of extracting the PSF on each image, registering it to a common reference frame and then using ALLFRAME to measure stellar magnitudes on each individual frame. In this case, we used the six best images (those with FWHM smaller than 0.13 arcsec) for a total exposure time of $1440 \mathrm{~s}$. We adopted this approach for the analysis of the external field (NGC 6388-b).

In both cases we find that the stellar PSF is better modeled with a quadratic dependence on the scientific frame, even though it is fairly uniform on the frame (up to a few percent), due to the excellent adaptive optics correction. We also evaluated all the possible expressions for the analytic part of the PSF, and selected the one giving the lowest chi-squared. The analytic profile that best fits the stellar PSF turned out to be a Penny function, i.e. a profile that has both a Gaussian core and power-law wings (Penny \& Dickens 1986). 


\section{Results}

As we discussed above, MAD observations were available only in the $K_{\mathrm{s}}$ band. In order to construct a CMD and ensure the largest possible color baseline, we used the $F 606 \mathrm{~W}$ ACS/HST photometry (Anderson et al. 2008) from the GO10775 Treasury project (P.I. Sarajedini). The ACS images cover both MAD fields. The photometric catalogue was produced by Anderson et al. (2008) using a dedicated procedure developed over the last few years (see also Anderson \& King 2000, 2006) and placed in the VEGAMAG system following Bedin et al. (2005) using the zeropoints of Sirianni et al. (2005). We also corrected the catalogs for the presence of differential reddening and possible zeropoint differences, using the empirical corrections described in Sarajedini et al. (2007); Milone et al. (2008).

Below, we will use observations of the external field of NGC 6388 to investigate the possible presence of multiple main sequences and/or SGBs. Because of the lower crowding, these images have a better photometric quality with respect to the central field ones, and a high level of photometric precision is needed to disentangle multiple populations in the CMD. We will use the central, more populous field to study the RGB and the HB morphology.

\subsection{External field (NGC 6388-b)}

In addition to being less crowded, the images of the external field also have better seeing. In particular, 6 out of 21 frames are characterized by a stellar FWHM of less than 0.13 arcsec. In order to maximize the precision of our photometry, we used these 6 best seeing images, for a total exposure time of $24 \mathrm{~min}$. We confirmed this decision by adding the remaining images, and noticing a degradation in the photometric quality.

We constructed the optical-NIR color-magnitude diagram by matching our stars with the ones measured on the ACS field. We obtained 7162 matches, and further selected them according to the parameters given by DAOPHOT, i.e. we excluded stars with a formal photometric error greater than 0.1 and with a $\chi^{2}$ greater than 5 . We also imposed the requirement that our matched stars have the same centroid within \pm 0.028 arcsec in both optical and NIR images. We excluded from the subsequent analysis stars found outside a circle having a radius of 800 pixels from the center of the image. This choice is motivated by the fact that the outermost region of the frame could be deteriorated because of non-optimal sky subtraction and, moreover, the outer regions could also be affected by imperfect distortion corrections. Therefore, the covered field is about $48 \times 48 \operatorname{arcsec}^{2}$. A total number of 2958 stars passed all the selection criteria. The optical-NIR CMD for them is shown in Fig. 3.

This is the deepest CMD available for this cluster in the NIR bands, and demonstrates that a ground-based 8-m class telescope, equipped with the appropriate $\mathrm{AO}$ correction, can give performance comparable to the ones reached by HST, thus nicely complementing the information at optical wavelengths.

Piotto (2008, see his Fig. 6) has shown that the SGB in the ACS CMD of NGC 6388 is divided into two branches, very similar to what has been identified in the SGB of NGC 1851 (Milone et al. 2008). In the top-left panel of Fig. 4 we show ACS/WFC photometry from Piotto (2008), on which we draw an estimated fiducial line that clearly separates the two SGB populations. The stars with a color between $\left(\left(m_{F 606 W}-m_{F 814 W}\right)=0.89\right.$ and 0.98 , and brighter than the fiducial line, are highlighted with red triangles (hereafter, according to Milone et al. 2008, notation we will refer to these stars as bright SGB, bSGB). The stars in the same

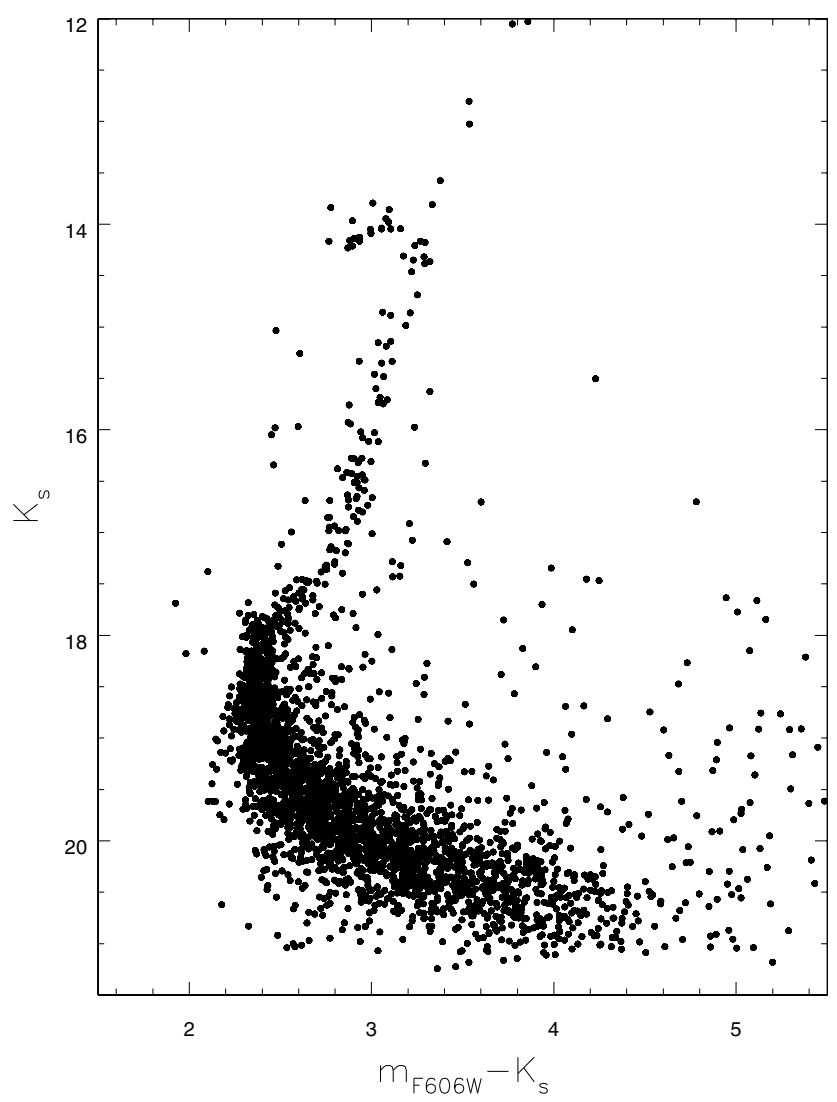

Fig. 3. Color-magnitude diagram of the external region of NGC 6388.

color interval that fell below the same fiducial line are marked with blue circles (faint SGB, fSGB).

In the top right panel of Fig. 4 we show - for the same stars the color-magnitude obtained combining MAD observations in $K_{\mathrm{S}}$ with ACS/WFC photometry in the $F 606 \mathrm{~W}$ filter.

When we plot the stars classified as bSGB and fSGB with the same symbols in this optical-NIR CMD, we can clearly see that bSGB are also systematically brighter than fSGB stars also in the $K_{\mathrm{s}}$ filter.

This is of particular relevance, since the absorption coefficient in the $K_{\mathrm{S}}$ band is $13 \%$ of the one present in the visible band (here $F 606 W)$, i.e. $0.37 \times E(B-V)$ (Schlegel et al. 1998) instead of $2.89 \times E(B-V)$ (Schlegel et al. 1998; Bedin et al. 2005; Sirianni et al. 2005; Girardi et al. 2008). Furthermore, we recall that we corrected the catalogs for differential reddening, as in Sarajedini et al. (2007), thus minimizing this effect. To better quantify the separation in $K_{\mathrm{s}}$ between bSGB and fSGB we first draw an estimated a fiducial line also in the optical-NIR CMD which follows the mean distribution of the two samples. Then we computed the difference $\Delta K \mathrm{~s}$ between each star, and the magnitude that a star with the same color would have if lying on this fiducial-line.

The two histograms shown in Fig. 4, lower panel, illustrate the results: we find a difference of 0.20 mag between the two peaks of the distributions (median values), with $\sigma=0.03$ and 0.05 for the upper and lower distributions, respectively. The distribution is clearly bimodal, indicating that two distinct populations are indeed present in NGC 6388.

In other words, the optical-NIR CMD of Fig. 4 reinforces the evidence that the SGB of NGC 6388 shows a split similar to that found in NGC 1851.

The KMM test (Ashman et al. 1994) reveals that a single Gaussian fit has to be discarded at the $93 \%$ level. 

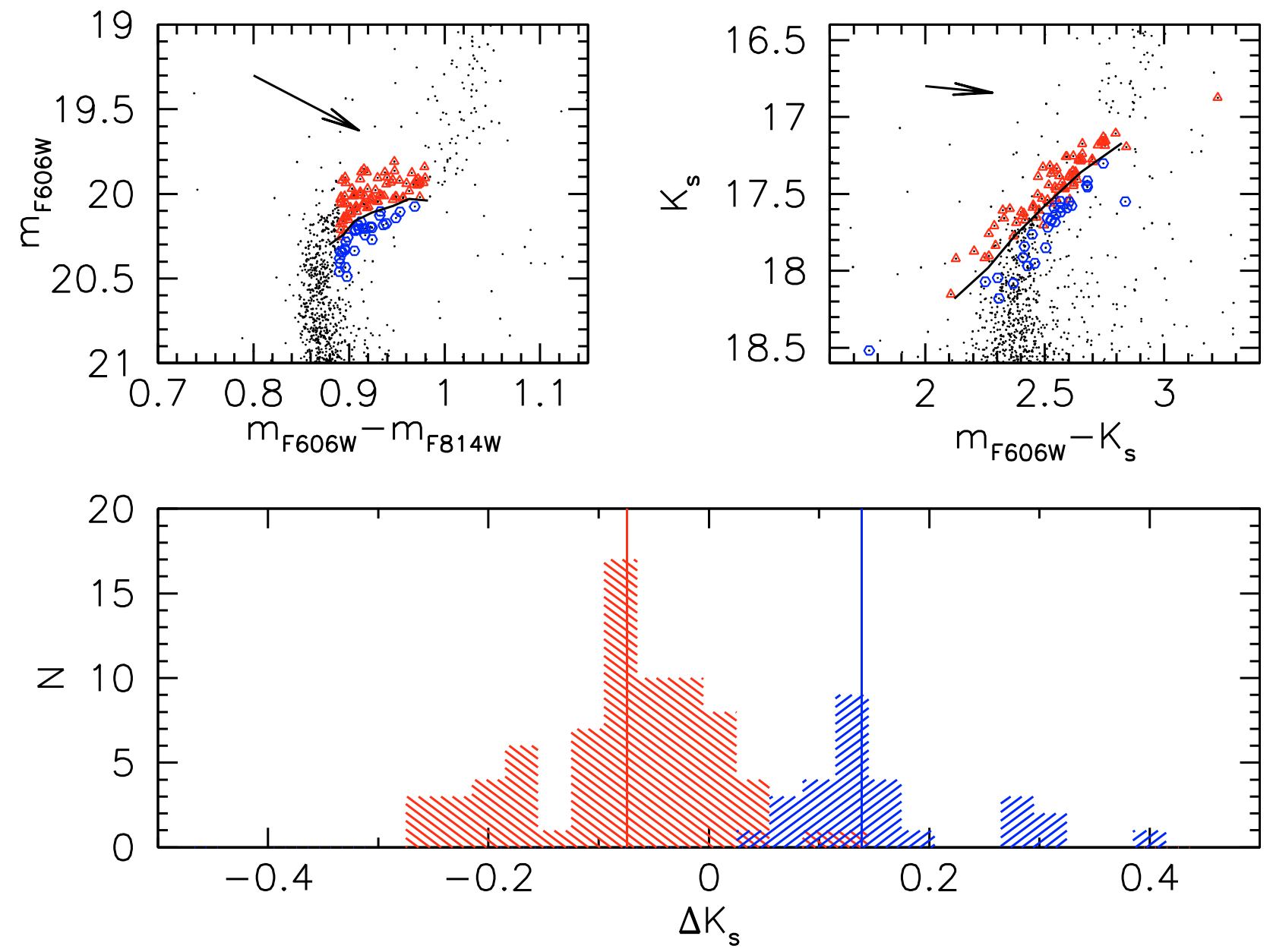

Fig. 4. Color-magnitude diagrams of the external region of NGC 6388. The upper left panel shows the pure optical CMD, with magnitudes coming from ACS/HST photometry, for the stars located in the MAD field: red triangles highlight stars on the bright SGB, blue open circles show the fainter SGB stars. The upper right panel shows the optical-NIR CMD. Red and blue dots represent the same stars identified in the upper left panel CMD. The lower panel shows the magnitude distribution of the two subpopulations with respect to a fiducial line drawn in the optical-NIR CMD.

We also performed a statistical analysis of the main sequence region, looking for evidence of double sequences. But the effort produced no result so far, due to the low precision of the photometry at the relatively faint level of the MS. Following the procedure described in Piotto et al. (2007), we calculated the color distribution of our selected stars and we fit them with Gaussian curves. The left panel of Fig. 5 shows a close-up of the opticalNIR CMD. In the middle panel we show the main sequence after subtraction of a fiducial line drawn by hand (the red continuous line in the left panel of Fig. 5). The rightmost panel shows the color distribution of the stars present in the middle panel, grouped into different magnitude bins. There is no evidence for multiple main sequences, a result that we also confirmed by using the KMM test (Ashman et al. 1994).

\subsection{Central field (NGC 6388-a)}

The central field of NGC 6388 suffers severe crowding. The sharp PSF provided by the AO correction makes crowding less of an issue.

We cannot directly compare our MAD photometry with other NIR CMDs (such as those obtained from SOFI or ISAAC), since we have observations in only one IR filter (the $K_{\mathrm{s}}$ band). But we can construct an optical-NIR CMD, which is shown in Fig. 6. In order to have the cleanest possible CMD, we excluded stars located outside a radius of 800 pixels from the center of the image. To pick the most isolated stars, we also excluded stars with a DAOPHOT error greater than 0.1. The number of selected stars is 1975. Because of the higher crowding, the CMD of the central field is much less deep than the one we obtained for the external region (see Sect. 3.1), even though the total exposure time of the analyzed frames is comparable (1000 s instead of $1440 \mathrm{~s}$ ). The left panel of Fig. 6 shows the $K_{\mathrm{s}}$ versus $m_{F 606 W}-K_{\mathrm{s}}$ CMD. Note that only the red clump in the RGB is visible: stars hotter (bluer) than the RR Lyrae gap are too faint in the $K$-band to be measured in our MAD images. Red encircled stars in Fig. 6 have been used to calculate the RGB luminosity functions (LF), which is shown in the right panels. The RGB bump is clearly visible both in the cumulative (upper right panel) and in the differential (lower right panel plot). The RGB bump and RGB tip are located at at $K_{\mathrm{s}}=14.30 \pm 0.05$ and $K_{\mathrm{s}}=8.95 \pm 0.05$, respectively. These values are in agreement with the ones given in Valenti et al. (2007), taking into account the errors.

\section{The age of NGC 6388}

We used our CMDs to estimate the age, reddening and distance of NGC 6388 using isochrone fitting. We used the isochrones by Pietrinferni et al. (2006), which are calculated for an $\alpha$-enhanced composition. Among the available metallicities, we used the 


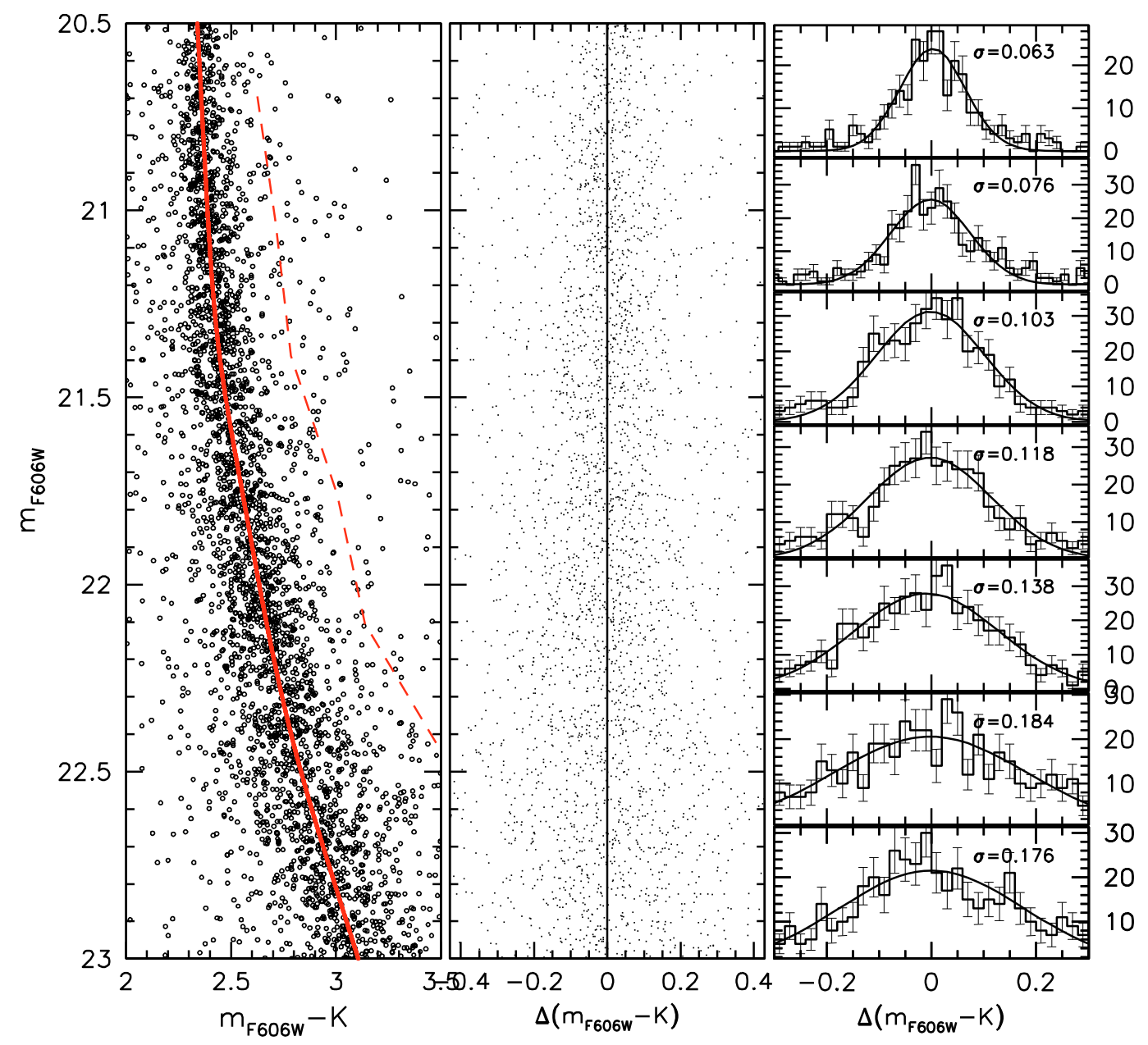

Fig. 5. Closeup of the main sequence region of the optical-infrared diagram. The left panel shows the fiducial line fitting the main sequence (drawn by hand); the dashed line represents the one $\sigma$ deviation. The middle panel shows the rectified CMD obtained by subtracting the color of the fiducial line from each star color; the right panel shows the histograms of color distribution of stars in magnitude bins and the Gaussian fitting profiles, together with an estimate of the $\sigma$ of the Gaussian.

isochrones for $[\mathrm{M} / \mathrm{H}]=-0.35$, which corresponds to $Z=0.0080$. We used the relations given in Schlegel et al. (1998) for the $K_{\mathrm{s}}$ extinction coefficient $\left[A_{K}=0.37 E(B-V)\right]$, while the absorption coefficient in $F 606 \mathrm{~W}\left[A_{F 606 W}=2.77 E(B-V)\right]$ has been taken from Bedin et al. (2005).

To begin, we used the $11.5 \mathrm{Gyr}$ isochrone to match the HBs of both the central and external fields and the MS stars of the external field with $19.0<m_{F 606 W}<20.0$ to fix the distance modulus and reddening (Figs. 7 and 8). In this way, we found $E(B-V)=0.47$ and $(m-M) 。=15.33$. Note that, in order to fit the RGB of the central field, we had to use the same distance modulus, but a lower reddening $E(B-V)=0.38$. Part of the reddening difference between the two fields is due to differential reddening. We note that Hughes et al. (2007) propose an even higher differential reddening than this. Still, we cannot exclude that photometric calibration errors can also contribute to the reddening difference. The distance modulus and the reddening for the central field are in good agreement with what was previously found by Valenti et al. (2007) and with the values given in the literature (see for instance the Harris 1996, online catalogue, which gives $E(B-V)=0.37)$.

As shown in Fig. 8, NGC 6388 has an age of 11.5 Gyr \pm 1.5 Gyr. The large dispersion reflects the dispersion of the stars along the SGB, as shown in the previous section. As discussed in Milone et al. (2008) and Cassisi et al. (2008) for the analogous case of NGC 1851, it is not clear at all that the SGB dichotomy can be interpreted in terms of an age difference only. A dichotomy in chemical composition (e.g. CNO content) could cause the morphology of the SGB in NGC 6388. In that case, the age difference between the two populations would be greatly reduced (Cassisi et al. 2008).

The average age we found is in agreement with many other NGC 6388 age determinations in the literature. Catelan et al. (2006) used HST observations and the $Q$ parameter to derive the cluster properties independently of the reddening and found an age similar to the age of 47 Tuc, with an error of 1 Gyr (e.g. $\sim 11 \pm 1$ Gyr, see Salaris \& Weiss 2002; Gratton et al. 2003; De Angeli et al. 2005). This would make our estimate of the cluster age compatible with the age determined so far. Hughes et al. (2007) derived an age of 12.6 Gyr from Washington photometry, also in agreement with our measurement. Yoon et al. (2008) reproduced the CMD of NGC 6388 derived by Rich et al. (1997) by using two populations of stars both having an age of 13 Gyr, but a different helium content (which they choose in order to be able to reproduce the blue HB).

\section{Conclusions}

We presented a pilot study that we carried out to investigate how well a MCAO/NIR system mounted on a $8 \mathrm{~m}$ telescope can 

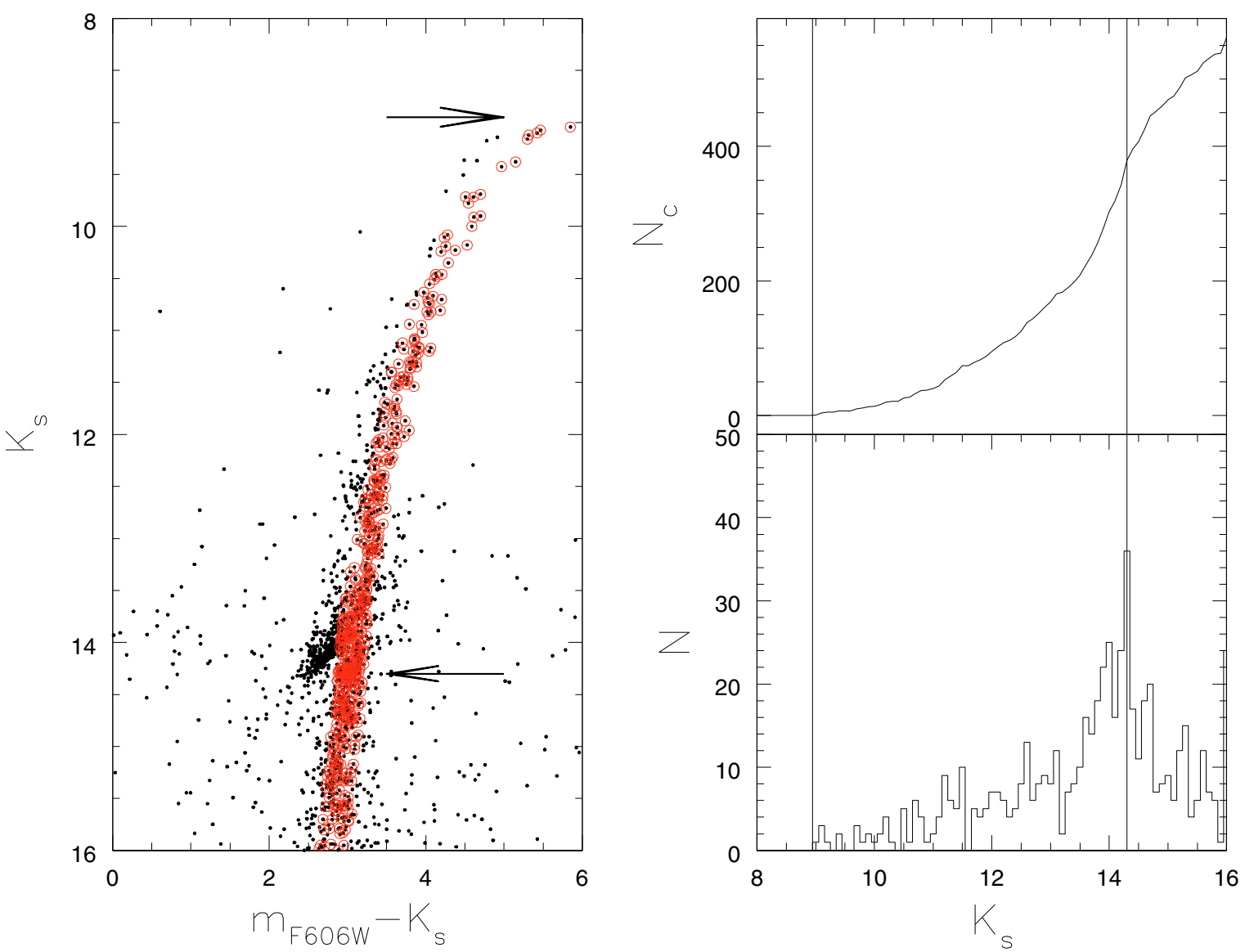

Fig. 6. Left panel: CMD of NGC 6388-a in $K_{\mathrm{s}}$. Right panel: cumulative (upper right panel) and differential (lower right panel) luminosity functions along the RGB. The two arrows show the positions of the RGB bump and tip.
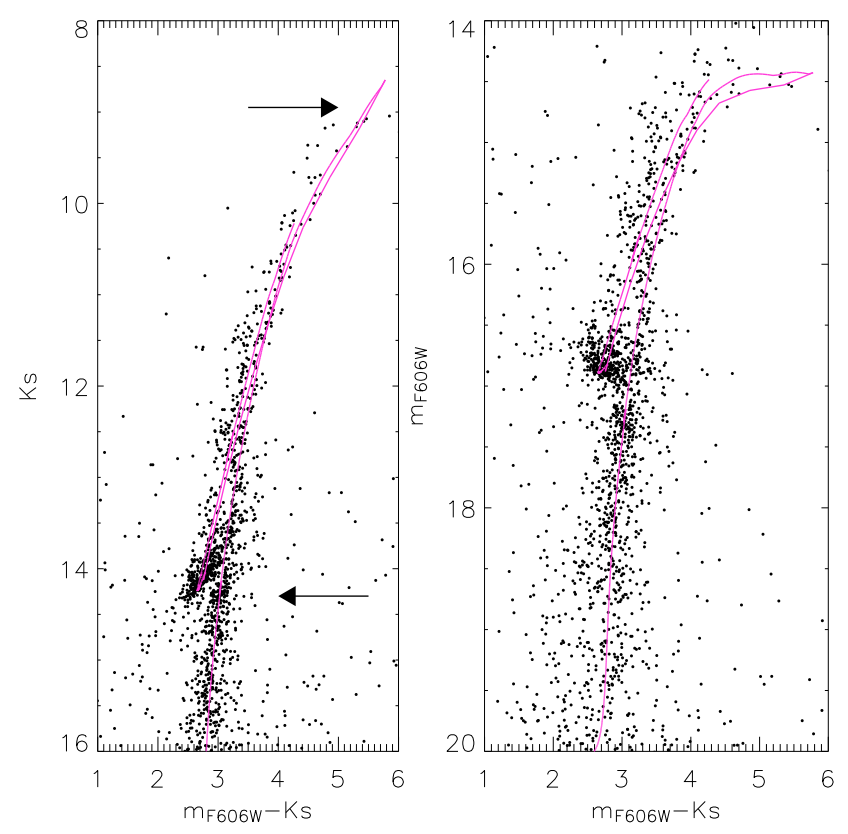

Fig. 7. Color-magnitude diagram of the central field of NGC 6388 in $K_{\mathrm{s}}$ versus $m_{F 606 W}-K_{\mathrm{s}}$ and $m_{F 606 W}$ versus $m_{F 606 W}-K_{\mathrm{s}}$. The superimposed isochrone has an age of $11.5 \mathrm{Gyr}$ and a metallicity of $[\mathrm{M} / \mathrm{H}]=-0.35$. The chemical composition is enhanced in $\alpha$-elements following the prescriptions given in Pietrinferni et al. (2006). The best fit of the isochrone implies a distance modulus of $(m-M) \circ=15.33$ and a reddening $E(B-V)=0.38$. perform photometry in crowded stellar field. We used the MultiConjugated Adaptive Optics Demonstrator (MAD) mounted on the VLT (Marchetti et al. 2007), coupled with the LayerOriented wavefront sensor (Ragazzoni et al. 2000; Arcidiacono et al. 2006, 2007), to observe in the $K_{\mathrm{s}}$ band two fields in the highly crowded Bulge GC NGC 6388. The morphology of the CMD of this cluster is still unexplained. We wanted to verify whether the recently suggested split in its SGB (first identified on ACS/HST images in optical bands) can also be seen in the NIR. Because of the high and differential reddening present in the cluster, NIR observations are particularly suited for our purposes.

By combining optical (ACS/HST) data with MAD $K_{\mathrm{s}}$ photometry, we obtained the deepest optical-nearIR CMD ever produced for NGC $6388^{1}$. The good photometric quality at the level of the SGB allowed us to construct a $K_{\mathrm{s}}$ vs. $m_{F 606 W}-K_{\mathrm{s}} \mathrm{CMD}$, which was previously seen by Piotto (2008) using only shortbaseline visible observations. The distribution of the stars along the SGB is clearly bimodal, indicating the presence of two different stellar populations. Whether this implies the presence of two groups of stars with a large age difference ( $>1$ Gyr), or a much smaller age difference but a dichotomy in chemical composition (e.g. CNO and helium) remains to be understood.

This study demonstrates the enormous potential of the new generation of MCAO instruments that will soon be available on large ground-based telescopes (LBT, E-ELT, etc.), and the

\footnotetext{
1 The photometric catalog will be available upon request to the authors.
} 

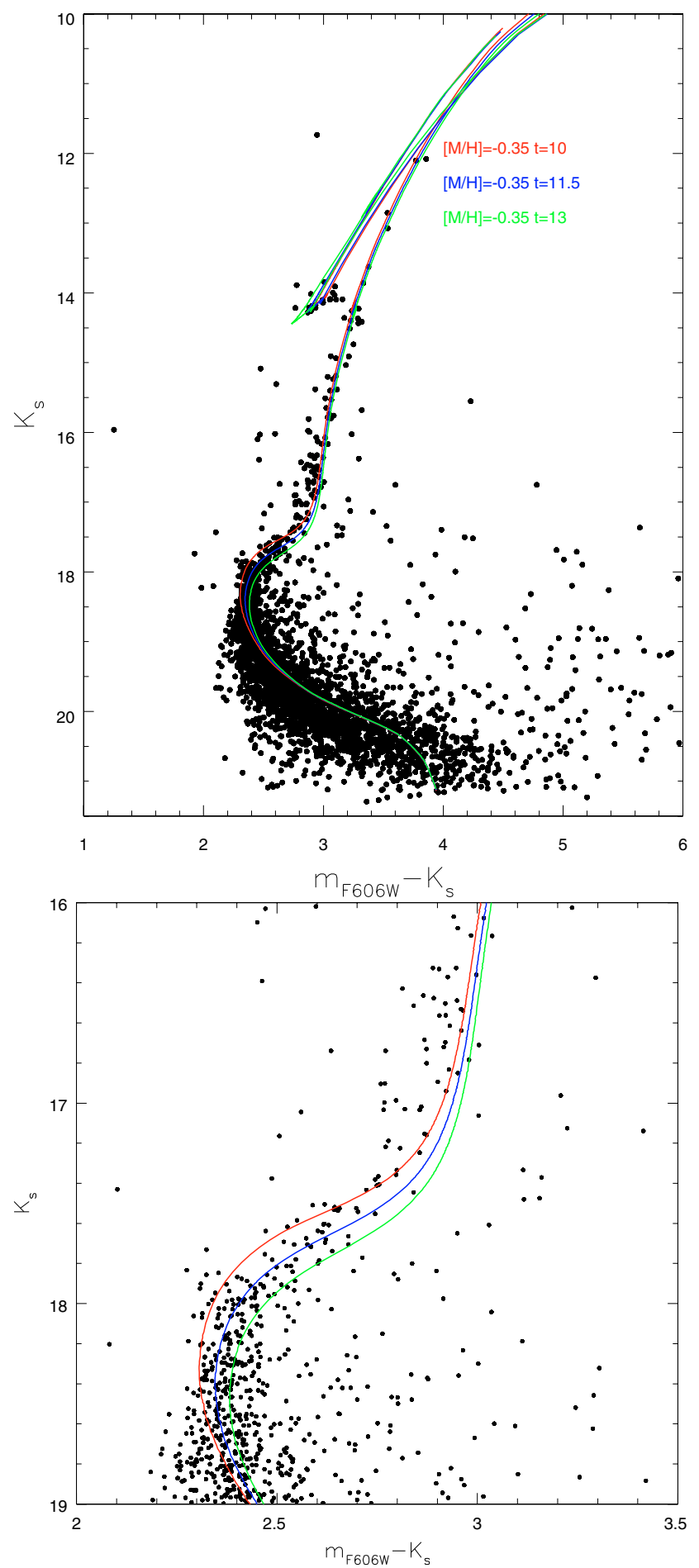

Fig. 8. Color-magnitude diagrams of the external field of NGC 6388 (NGC 6388-b). The superimposed isochrones have ages of 10, 11.5 and $13 \mathrm{Gyr}$ (red, blue, and green, respectively) and a metallicity of $[\mathrm{M} / \mathrm{H}]=-0.35, \alpha$-enhanced following the prescriptions given in Pietrinferni et al. (2006). The best fit of the HB luminosity and of the color of the main sequence stars with $19.0<K_{\mathrm{s}}<20.0$ implies a distance modulus of $(m-M) \circ=15.33$ and $E(B-V)=0.47$. The lower panel shows a zoom around the SGB region.

advantage of coupling high-resolution optical images from space with ground-based AO-corrected NIR data.

Acknowledgements. Based on observations obtained with the MCAO Demonstrator at the VLT Melipal Nasmyth focus. We wish to thank P. Amico for her support during the observations, Y. Momany for his helpful advice and S. Cassisi for the set of helium-enriched isochrones. We also thank the anonymous referee for the careful reading of the manuscript, and for the useful comments.

\section{References}

Anderson, J., \& King, I. R. 2000, PASP, 112, 1360

Anderson, J., \& King, I. R. 2006, PSFs, Photometry, and Astronomy for the ACS/WFC, Tech. rep.

Anderson, J., Sarajedini, A., Bedin, L. R., et al. 2008, AJ, 135, 2055

Arcidiacono, C., Lombini, M., Diolaiti, E., Farinato, J., \& Ragazzoni, R. 2006, in Advances in Adaptive Optics II, ed. B. L. Ellerbroek, \& C. D. Bonaccini, Proc. SPIE, 6272, 627227

Arcidiacono, C., Lombini, M., Farinato, J., \& Ragazzoni, R. 2007, Mem. Soc. Astron. Ital., 78, 708

Ashman, K. M., Bird, C. M., \& Zepf, S. E. 1994, AJ, 108, 2348

Bedin, L. R., Piotto, G., Anderson, J., et al. 2004, ApJ, 605, L125

Bedin, L. R., Cassisi, S., Castelli, F., et al. 2005, MNRAS, 357, 1038

Busso, G., Cassisi, S., Piotto, G., et al. 2007, A\&A, 474, 105

Caloi, V., \& D’Antona, F. 2007, A\&A, 463, 949

Carretta, E., Recio-Blanco, A., Gratton, R. G., Piotto, G., \& Bragaglia, A. 2007, ApJ, 671, L125

Cassisi, S., Salaris, M., Pietrinferni, A., et al. 2008, ApJ, 672, L115

Catelan, M., Stetson, P. B., Pritzl, B. J., et al. 2006, ApJ, 651, L133

D’Antona, F., \& Caloi, V. 2004, ApJ, 611, 871

D’Antona, F., \& Caloi, V. 2008, in IAU Symp., 246, 156

D’Antona, F., Caloi, V., Montalbán, J., Ventura, P., \& Gratton, R. 2002, A\&A, 395, 69

De Angeli, F., Piotto, G., Cassisi, S., et al. 2005, AJ, 130, 116

Falomo, R., Pian, E., Treves, A., et al. 2008, A\&A, in prep.

Ghedina, A., Cecconi, M., Ragazzoni, R., et al. 2003, in Adaptive Optical System

Technologies II, ed. P. L. Wizinowich, \& D. Bonaccini, Proc. SPIE, 4839, 869

Girardi, L., Dalcanton, J., Williams, B., et al. 2008, PASP, 120, 583

Gratton, R. G., Bragaglia, A., Carretta, E., et al. 2003, A\&A, 408, 529

Gullieuszik, M., Greggio, L., Held, E. V., et al. 2008, A\&A, 483, L5

Harris, W. E. 1996, AJ, 112, 1487

Hawarden, T. G., Leggett, S. K., Letawsky, M. B., Ballantyne, D. R., \& Casali, M. M. 2001, MNRAS, 325, 563

Hughes, J., Wallerstein, G., Covarrubias, R., \& Hays, N. 2007, AJ, 134, 229

Lee, Y.-W., Joo, J.-M., Sohn, Y.-J., et al. 1999, Nature, 402, 55

Marchetti, E., Brast, R., Delabre, B., et al. 2006, in Advances in Adaptive Optics

II, ed. B. L. Ellerbroek, \& C. D. Bonaccini, Proc. SPIE, 6272

Marchetti, E., Brast, R., Delabre, B., et al. 2007, The Messenger, 129, 8

Milone, A. P., Bedin, L. R., Piotto, G., et al. 2008, ApJ, 673, 241

Pancino, E., Ferraro, F. R., Bellazzini, M., Piotto, G., \& Zoccali, M. 2000, ApJ, 534, L83

Penny, A. J., \& Dickens, R. J. 1986, MNRAS, 220, 845

Pietrinferni, A., Cassisi, S., Salaris, M., \& Castelli, F. 2006, ApJ, 642, 797

Piotto, G. 2008, Mem. Soc. Astron. Ital., 79, 334

Piotto, G., Bedin, L. R., Anderson, J., et al. 2007, ApJ, 661, L53

Ragazzoni, R. 1996, J. Mod. Opt., 43, 289

Ragazzoni, R., \& Farinato, J. 1999, A\&A, 350, L23

Ragazzoni, R., Farinato, J., \& Marchetti, E. 2000, in Adaptive Optical Systems Technology, ed. P. L. Wizinowich, Proc. SPIE, 4007, 1076

Raimondo, G., Castellani, V., Cassisi, S., Brocato, E., \& Piotto, G. 2002, ApJ, 569,975

Ree, C. H., Yoon, S.-J., Rey, S.-C., \& Lee, Y.-W. 2002, in Omega Centauri, A Unique Window into Astrophysics, ed. F. van Leeuwen, J. D. Hughes, \& G. Piotto, ASP Conf. Ser., 265, 101

Rich, R. M., Minniti, D., \& Liebert, J. 1993, ApJ, 406, 489

Rich, R. M., Sosin, C., Djorgovski, S. G., et al. 1997, ApJ, 484, L25

Salaris, M., \& Weiss, A. 2002, A\&A, 388, 492

Sarajedini, A., Bedin, L. R., Chaboyer, B., et al. 2007, AJ, 133, 1658

Schlegel, D. J., Finkbeiner, D. P., \& Davis, M. 1998, ApJ, 500, 525

Sirianni, M., Jee, M. J., Benítez, N., et al. 2005, PASP, 117, 1049

Stetson, P. B. 1987, PASP, 99, 191

Valenti, E., Ferraro, F. R., \& Origlia, L. 2007, AJ, 133, 1287

Vernet, E., Arcidiacono, C., Baruffolo, A., et al. 2005, Opt. Eng., 6601

Villanova, S., Piotto, G., King, I. R., et al. 2007, ArXiv Astrophysics e-prints

Yoon, S.-J., Joo, S.-J., Ree, C. H., et al. 2008, ApJ, 677, 1080 